\title{
Validation of an HPLC Method for the Determination of Valdecoxib and its Degradation Product: a Mixture of $\alpha$ - and $\beta$-n-Lactosyl Sulfonamide Anomers
}

\author{
G. Savić ${ }^{1}$, M. Zečević ${ }^{2, 凶}$, B. Jocić ${ }^{2}$, Li. Živanović ${ }^{2}$
}

${ }^{1}$ Medicines and Medical Devices Agency of Serbia, Vojvode Stepe 458, 11152 Belgrade, Serbia

2 Institute of Pharmaceutical Chemistry and Drug Analysis, Faculty of Pharmacy, Vojvode Stepe 450,

P.O. Box 146, 11000 Belgrade, Serbia; E-Mail: mzecevic@pharmacy.bg.ac.yu

\section{Erratum to: Chromatographia 66(1-2):29-35 DOI 10.1365/s10337-007-0276-9}

Unfortunately, in the original article the acknowledgement part is missing:

Acknowledgement

These results are part of the project "Synthesis, Quantitative Structure/Properties and Activity Relationship, Physical-Chemical Characterization and Analysis of Pharmacologically Active Substances" No. 142071 B, financed by the Ministry of Science and Technology of the Republic of Serbia. 